\title{
One-neutron knockout from light neutron-rich nuclei at relativistic energies
}

\author{
C. Rodríguez-Tajes, ${ }^{1,{ }^{*}}$ H. Álvarez-Pol, ${ }^{1}$ T. Aumann, ${ }^{2}$ E. Benjamim,,${ }^{1}$ J. Benlliure,${ }^{1}$ M. J. G. Borge,${ }^{3}$ M. Caamaño, ${ }^{1}$ \\ E. Casarejos,,${ }^{1, \dagger}$ A. Chatillon, ${ }^{2}$ D. Cortina-Gil,${ }^{1}$ K. Eppinger, ${ }^{4}$ T. Faestermann,${ }^{4}$ M. Gascón, ${ }^{1}$ H. Geissel,,${ }^{2}$ R. Gernhäuser, ${ }^{4}$ \\ B. Jonson, ${ }^{5,6}$ R. Kanungo,${ }^{7}$ R. Krücken, ${ }^{4}$ T. Kurtukian,,${ }^{1,+}$ K. Larsson, ${ }^{5}$ P. Maierbeck,${ }^{4}$ T. Nilsson,,${ }^{5}$ C. Nociforo, ${ }^{2}$ \\ C. Pascual-Izarra, ${ }^{3}$ A. Perea, ${ }^{3}$ D. Pérez-Loureiro, ${ }^{1}$ A. Prochazka, ${ }^{2}$ S. Schwertel, ${ }^{4}$ H. Simon, ${ }^{2}$ K. Sümmerer, ${ }^{2}$ O. Tengblad, ${ }^{3}$ \\ H. Weick, ${ }^{2}$ M. Winkler, ${ }^{2}$ and M. Zhukov ${ }^{5}$ \\ ${ }^{1}$ Universidade de Santiago de Compostela, E-15782 Santiago de Compostela, Spain \\ ${ }^{2}$ GSI Helmholtzzentrum für Schwerionenforschung, D-64291 Darmstadt, Germany \\ ${ }^{3}$ Instituto de Estructura de la Materia, CSIC, E-28006 Madrid, Spain \\ ${ }^{4}$ Physik Department E12, Technische Universität München, D-85748 Garching, Germany \\ ${ }^{5}$ Fundamental Fysik, Chalmers Tekniska Högskola, S-412 96 Göteborg, Sweden \\ ${ }^{6}$ PH Department, CERN, 1211 Geneve CH-23, Switzerland \\ ${ }^{7}$ Astronomy and Physics Department, Saint Mary's University, Halifax, Nova Scotia B3H 3C3, Canada \\ (Received 18 April 2010; revised manuscript received 20 July 2010; published 5 August 2010; corrected 30 August 2010)
}

\begin{abstract}
One-neutron knockout reactions from neutron-rich nuclei, with $Z=6-13$ and $N=8-22$, were studied at the Fragment Separator (GSI) at high beam energies, around $700 \mathrm{MeV} /$ nucleon. Structural phenomena such as the formation of one-neutron halos in odd-mass carbon isotopes $\left({ }^{15,17,19} \mathrm{C}\right)$ will be discussed. In addition, one-neutron knockout measurements from ${ }^{22} \mathrm{~N}$ were carried out for the first time and demonstrate clearly the change from a $0 d_{5 / 2}$ to a $1 s_{1 / 2}$ orbital for the valence neutron, an effect that is expected above $N=14$ and that was also observed in ${ }^{23} \mathrm{O}$ and ${ }^{24} \mathrm{~F}$. The possibility of an anomalous structure of ${ }^{26} \mathrm{~F}$, due to a significant $1 s_{1 / 2}$ neutron admixture, will also be discussed in the light of the experimental data obtained in this work. Finally, the ground-state configuration of neutron-rich neon isotopes $\left({ }^{24-28} \mathrm{Ne}\right)$ was studied, providing new information in a region that is relatively close to the island of inversion.
\end{abstract}

DOI: 10.1103/PhysRevC.82.024305 PACS number(s): 21.10.Gv, 21.10.Pc, 21.60.Cs, 24.50.+g

\section{INTRODUCTION}

In the last decades, experimental research with radioactive beams, in conjunction with new theoretical developments, has provided new insights on the evolution of the shell structure when moving away from the valley of beta stability and has led to the discovery of new, unexpected, and exciting effects.

Much interest has been directed toward halo nuclei, characterized by a low-density tail in the wave function of the valence nucleon(s), which extends beyond the standard nuclear radius [1]. Several halo nuclei were found near the neutron drip line and new candidates were suggested [2]. An example is ${ }^{22} \mathrm{~N}$, for which an enlarged spatial distribution of the $1 s_{1 / 2}$ neutron state was proposed [3] and might be supported by recent spectroscopic data [4].

Experiments have also revealed significant differences with respect to the magic numbers established for stable nuclei such as the vanishing of the $N=8$ closed shell for ${ }^{12} \mathrm{Be}[5,6]$ or the appearance of new subshell closures at $N=14$ and 16 for oxygen isotopes [3,7-12].

In addition to this, a breakdown of the conventional shell closure at $N=20$ was observed for very neutron-rich nuclei. The first indication of this effect arrived from mass

\footnotetext{
*carme.rodriguez@usal.es; Present address: Centro de Láseres Pulsados Ultracortos Ultraintensos, 37008 Salamanca, Spain.

†Present address: Universidade de Vigo, 36310 Vigo, Spain.

${ }^{\ddagger}$ Present address: Centre d’Études Nucléaires de Bordeaux Gradignan, 33175 Gradignan, France.
}

measurements of ${ }^{31,32} \mathrm{Na}$, which showed that these nuclei are more deeply bound than expected in the light of the $N=20$ shell closure [13]. This area of the nuclear chart around $N=20$ is commonly called the island of inversion because the ground states are believed to consist of intruder configurations with neutrons promoted from the $s d$ to the $p f$ shell, as it was proposed in the earlier work of Ref. [14]. Later on, evidences of deformation emerged for ${ }^{32} \mathrm{Mg}[15],{ }^{30} \mathrm{Ne}$ [16], and others. The proximity of neutron-rich neon isotopes to this region makes their investigation especially attractive. SDPF-M calculations [17], which allow the mixing of $s d$ and $p f$ configurations, are needed to reproduce the $3 / 2^{-}$level of ${ }^{27} \mathrm{Ne}$ at $765 \mathrm{keV}[18-20]$ and the first excited state of ${ }^{28} \mathrm{Ne}$, located at $1293 \mathrm{keV}$, with $I^{\pi}=2^{+}[18,21]$. The importance of intruder configurations in the ground state of ${ }^{28} \mathrm{Ne}$ was also shown in an earlier one-neutron knockout experiment in which accurate spectroscopic measurements were carried out [20]. More recent information about the ground state of this nucleus can be found in Ref. [22], where the major role of excited states of the core is confirmed.

Breakup reactions, with cross sections that can amount to more than $100 \mathrm{mb}$ for weakly bound nuclei, constitute an experimental technique suitable for the investigation of structural phenomena as those described previously. In particular, the interest in one-nucleon knockout reactions was triggered in the mid-1980s by the study of halo nuclei [23-29]. In the context of one-neutron halos, the weak binding of the valence neutron causes a significant increase of the one-neutron knockout cross section and the emerging $A-1$ fragments exhibit narrow momentum distributions that reflect the spatial extension of the halo. 
With time, the knockout technique has extended to other exotic species. In general, the shape and width of the $A-1$ fragment momentum distributions are sensitive to the orbital angular momentum of the neutron removed in the reaction and can be used to explore the ground-state structure of the projectiles [30], understood as the contribution of several core $\otimes$ neutron configurations.

One-neutron knockout reactions were used in this experiment to investigate the ground-state structure of 39 neutron-rich projectiles, covering the region $Z=6-13$ and $N=8-22$. The use of a beryllium target ensured a negligible contribution from Coulomb dissociation. Moreover, the transparent limit of the Serber model [31] is valid in this high energy regime (700 MeV/nucleon), which means that the one-neutron knockout channel is dominated by stripping reactions, where the last neutron is absorbed by the target and the $A-1$ fragment remains unaffected by the process. The study of the $A-1$ fragment provides meaningful information about the neutron removed in the reaction.

\section{EXPERIMENT}

The experiment was performed using the Fragment Separator (FRS) [32], a high-resolution magnetic spectrometer installed at GSI (Darmstadt, Germany) [33]. A fully ionized ${ }^{40} \mathrm{Ar}$ primary beam was accelerated in the SIS heavy ion synchrotron up to $700 \mathrm{MeV} /$ nucleon and directed toward a $4 \mathrm{~g} / \mathrm{cm}^{2}$ Be target located at the entrance of the FRS, where a cocktail of exotic neutron-rich nuclides was produced via fragmentation reactions. The mean intensity was around $10^{10} \mathrm{Ar}$-ions/spill, for spills with a duration of 4-6 s.

Figure 1 shows a schematic view of the detector arrangement in the FRS. The neutron-rich isotopes were separated and identified in the first half of the FRS (F0-F2). Then, the one-neutron knockout reaction took place at the intermediate focal plane (F2), in a $1720 \mathrm{mg} / \mathrm{cm}^{2}$ Be target. The analysis of the knockout fragments was performed in the second part of the FRS (F2-F4).

The experimental setup allowed a clean selection of the reaction channel based on the identification of both the

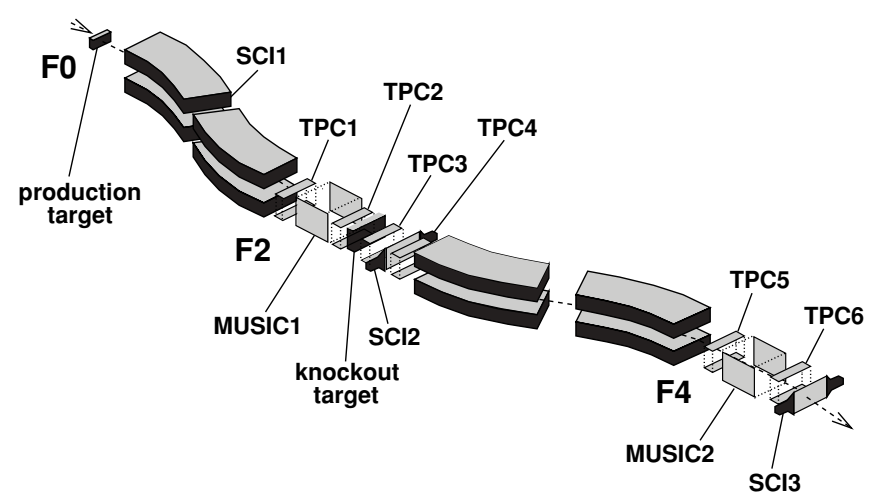

FIG. 1. Experimental setup in the FRS. The detectors were concentrated at the intermediate and final focal planes, labeled F2 and F4. The charges of the nuclei were measured with MUSIC. Their velocities were obtained from time-of-flight measurements provided by the scintillators (SCI) and their positions at the intermediate and final focal planes were given by time-projection chambers (TPC).

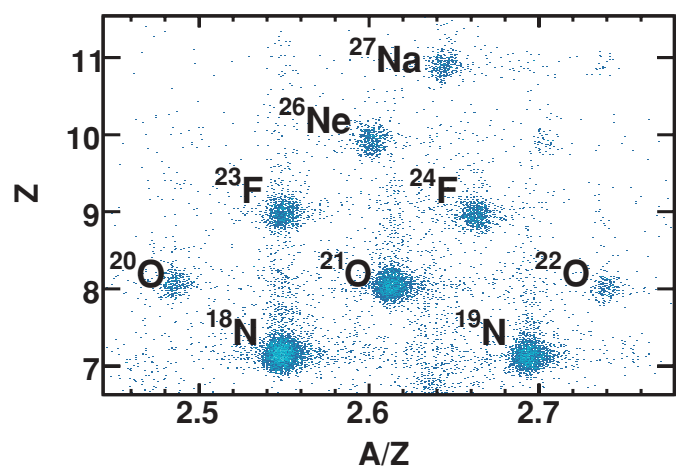

FIG. 2. (Color online) Identification of incoming projectiles at the FRS intermediate focal plane, the charge is represented against the mass-to-charge ratio. A similar graph was obtained for the reaction products at the final focal plane, ensuring a clean selection of the reaction channel.

projectile and the one-neutron knockout fragment. For that purpose, the charges of the fully ionized nuclei were measured in multiple-sample ionization chambers (MUSIC) [34]. To distinguish between different isotopes, the mass-to-charge ratio $m_{0} / q$ was also determined. This quantity can easily be determined through the magnetic rigidity $B \rho=\gamma m_{0} \beta c / q$, which was obtained from position measurements in the six time-projection chambers (TPC1-6) [35] located around the FRS focal planes. Additional measurements of the velocity $\beta$ from the time-of-flight between plastic scintillator pairs (SCI1-SCI2 and SCI2-SCI3) were used to finally calculate the mass-to-charge ratio. An example of the quality of the separation between the produced nuclei is shown in Fig. 2 .

A double condition that selected the projectile and the fragment of interest was used to determine the one-neutron knockout cross section. To properly evaluate the number of one-neutron knockout events, the transmission of the reaction products through the FRS was accounted for by means of MOCADI simulations [36], where the detector setup and the optics used in this experiment were carefully reproduced to reconstruct the flight of each nucleus along the spectrometer. The contribution of those reactions that did not take place in the beryllium target located at F2, but in the materials surrounding it, such as the plastic scintillator, should also be accounted for. This task required dedicated sets of measurements with an empty target, which could only be performed for a reduced number of cases, namely, ${ }^{16,17} \mathrm{C},{ }^{18-20} \mathrm{~N},{ }^{20-22} \mathrm{O},{ }^{23-25} \mathrm{~F}$, ${ }^{26,27} \mathrm{Ne},{ }^{29,30} \mathrm{Na}$, and ${ }^{31,32} \mathrm{Mg}$ one-neutron knockout.

The second physical observable determined in this experiment was the longitudinal-momentum distribution of the one-neutron knockout fragments, which is known from experiments and theory to be less affected by the reaction mechanism than the components transverse to the beam direction and is then regarded as a better probe to explore the projectile structure $[37,38]$. In the context of the transparent limit of the Serber model, the longitudinal-momentum distribution of the one-neutron knockout fragments can be identified with the ground-state distribution of the removed neutron inside the projectiles. The width of the longitudinalmomentum distribution is noticeably narrower for $l=0$ than 


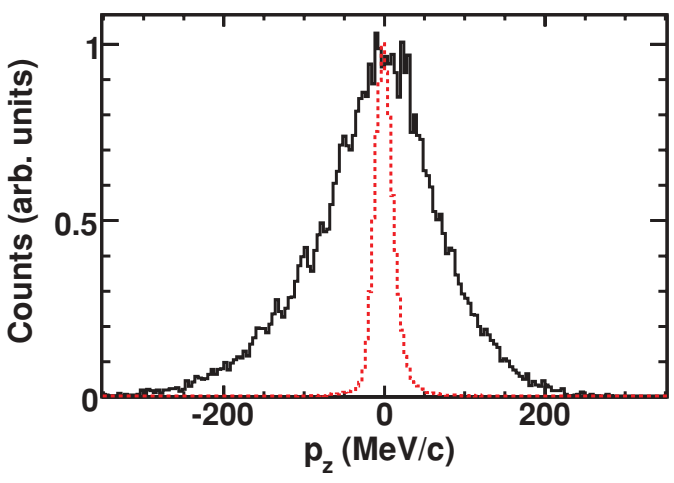

FIG. 3. (Color online) Dotted line: ${ }^{24} \mathrm{~F}$ projectiles that did not react in the intermediate focal plane of the FRS, FWHM $=26 \pm$ $2 \mathrm{MeV} / \mathrm{c}$. Solid line: ${ }^{23} \mathrm{~F}$ fragments after one-neutron knockout of ${ }^{24} \mathrm{~F}, \mathrm{FWHM}=158 \pm 8 \mathrm{MeV} / \mathrm{c}$. The width of this distribution was corrected for the momentum resolution, giving a final value of $156 \pm$ $8 \mathrm{MeV} / \mathrm{c}$. Similar procedures were applied to all the cases studied in this experiment.

for $l=2$ and can be used to identify the orbital angular momentum of the valence neutron. However, it is important to note that the core survival implies that only the outer part of the wave function is sampled with this technique $[39,40]$.

The longitudinal-momentum distributions were obtained in the reference frame of the laboratory from position measurements at the intermediate and final focal planes, using the transfer matrix of the FRS optical system. Afterward, a Lorentz transformation was applied to convert them into the co-moving frame of the projectile.

The widths of the longitudinal-momentum distributions were determined from Gaussian fits in the central regions, minimizing effects due to the acceptance limits of the spectrometer. The momentum resolution, which depends on several factors such as the resolution of the spectrometer, the optical quality of the primary beam, the amount of matter in the midplane and the resolution of the detectors, was determined from nonreacting projectiles that reached the final focal plane. This correction is illustrated in Fig. 3 and reduced the widths obtained for the one-neutron knockout fragments by $1-5 \%$.

\section{RESULTS}

The longitudinal-momentum distributions measured in this work are shown in Figs. 4 and 5, where the Gaussian fits used to extract the values of their widths are also indicated. It should be noted that, in the case of ${ }^{15} \mathrm{C}$ one-neutron knockout, the distribution is noticeably distorted due to the acceptance limits of the FRS and a graphical evaluation of its width was applied. These results, properly corrected for the experimental momentum resolution, are summarized in Table I, which also gives the full width at half maximum (FWHM) values available in the literature and includes the one-neutron knockout cross sections measured in this experiment.

To effectively treat such a significant amount of data, the discussion of the results will be organized around specific topics of interest and will focus on certain isotopic chains, namely, ${ }^{14-19} \mathrm{C},{ }^{16-22} \mathrm{~N},{ }^{19-23} \mathrm{O},{ }^{21-26} \mathrm{~F}$, and ${ }^{24-28} \mathrm{Ne}$.

\section{A. Results from ${ }^{14-19} \mathrm{C}$}

The one-neutron knockout cross sections and longitudinalmomentum distributions measured in this work are represented in Fig. 6 as a function of the neutron number in the projectile and will be used here to discuss the appearance of one-neutron halos in ${ }^{15,17,19} \mathrm{C}$ isotopes.

A narrowing of the longitudinal-momentum distribution was observed for ${ }^{14} \mathrm{C}$ fragments produced in the one-neutron knockout of ${ }^{15} \mathrm{C}$ projectiles, reflecting the dominance of a $1 s_{1 / 2}$ intruder configuration. Such a behavior, combined with a clear increase of the cross section and a relatively low binding energy of the last neutron $\left(S_{n}=1218.1 \pm 0.8 \mathrm{keV}\right.$ [41]), can be understood in the light of a halo-like structure dominated by a $1 s_{1 / 2}$ valence neutron coupled to the $0^{+}$ground state of ${ }^{14} \mathrm{C}$, which was already suggested in other works [42,47-49] and is supported by spectroscopic and quenching factors calculated from ${ }^{15} \mathrm{C}$ one-neutron knockout in Ref. [50].

The heavier odd-mass isotope ${ }^{17} \mathrm{C}$ has, despite its low one-neutron separation energy $\left(S_{n}=727 \pm 18 \mathrm{keV}\right.$ [41]), a broad momentum distribution and a smaller one-neutron cross section, which imply no halo formation. The enhanced width of the momentum distribution indicates an $l=2$ nature of the valence neutron, in good agreement with the work of Ref. [27], where the authors found that the ground state of ${ }^{17} \mathrm{C}$ is dominated by a ${ }^{16} \mathrm{C}\left(2^{+}\right) \otimes 0 d_{5 / 2}$ component. Such a configuration, where a $0 d_{5 / 2}$ neutron is coupled to the first $2^{+}$state of ${ }^{16} \mathrm{C}$, at $1766 \mathrm{keV}$, is associated with a higher effective-separation energy and an increase of the centrifugal barrier, which explains the observed halo suppression.

The structure of ${ }^{19} \mathrm{C}$ has attracted much attention due to its remarkably low one-neutron separation energy $\left(S_{n}=\right.$ $577 \pm 94 \mathrm{keV}$ [41]), which will result in a well-developed one-neutron halo if the valence neutron occupies an $s$ or $p$ orbital. The cross section measured in this work is clearly increased with respect to the neighboring isotopes and the width of the fragment momentum distribution undergoes a reduction by a factor larger than two. Both observations can be interpreted as a reliable signature of a halo configuration and indicate an $l=0$ character of the valence neutron, confirming the conclusions of earlier experiments [27,51,52].

Very interesting calculations in the framework of the eikonal model were performed within the last years and allowed to successfully reproduce experimental one-neutron knockout cross sections of neutron-rich carbon isotopes at energies about $100 \mathrm{MeV} /$ nucleon (see Ref. [53] and references therein). This work extends the available one-neutron knockout measurements to higher energies, and although a direct comparison should be taken cautiously due to the different energy regimes and the importance of empty target corrections reported in this experiment, a fair agreement in the trend of the data is observed.

\section{B. Results for ${ }^{16-22} \mathrm{~N},{ }^{19-23} \mathrm{O},{ }^{21-26} \mathrm{~F}$}

The results concerning ${ }^{16-22} \mathrm{~N},{ }^{19-23} \mathrm{O}$, and ${ }^{21-26} \mathrm{~F}$ projectiles are displayed in Fig. 7. A clear effect becomes visible above $N=14$, with a smaller width of the fragment longitudinal-momentum distribution and a larger one-neutron knockout cross section. Such a behavior can be understood 

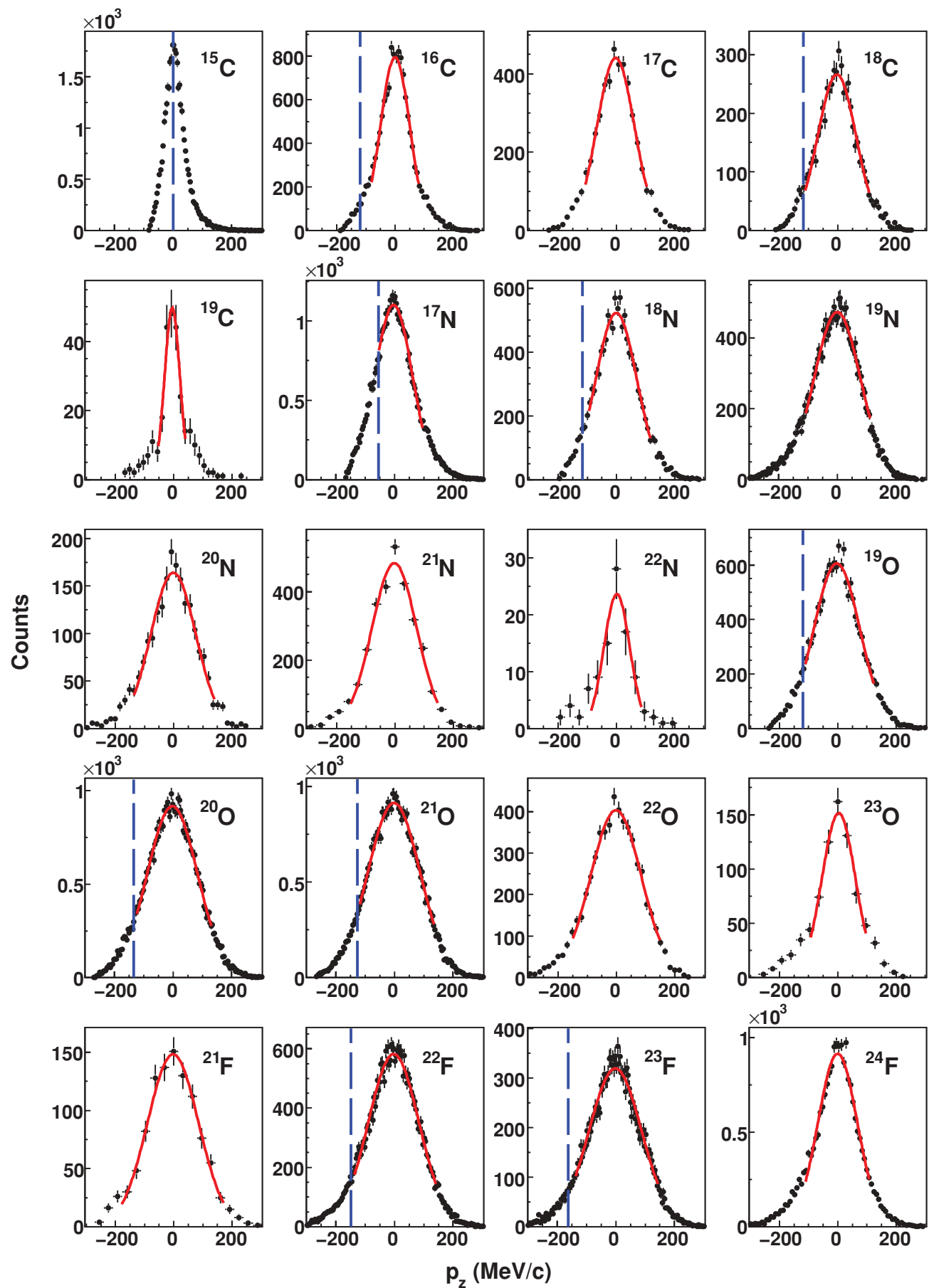

FIG. 4. (Color online) Fragment longitudinal-momentum distributions measured after one-neutron knockout of ${ }^{15-19} \mathrm{C}$, ${ }^{17-22} \mathrm{~N}$, ${ }^{19-23} \mathrm{O}$, and ${ }^{21-24} \mathrm{~F}$ projectiles. The solid lines represent the Gaussian fits used to determine the widths of the distributions and the vertical dashed lines indicate negative-momentum regions affected by the acceptance limits of the spectrometer.

within a simple shell-model picture. For neutron numbers $N=8$ to 14 , the valence neutrons will occupy a $0 d_{5 / 2}$ orbit, while, for $N=15$ to 16 , they will stay in a $1 s_{1 / 2}$ level. This change from the $l=2$ to $l=0$ configurations explains the narrowing of the momentum distributions. The enhanced cross sections can be understood as a direct consequence of the weaker binding energies and significant $1 s_{1 / 2}$ admixtures, where the core levels are completely filled up to $0 d_{5 / 2}$ and there is a single neutron in a $1 s_{1 / 2}$ orbit. This effect was reported earlier for ${ }^{23} \mathrm{O}$ and ${ }^{24} \mathrm{~F}$ in Refs. [44,49].

Special attention should be dedicated to ${ }^{22} \mathrm{~N}$, which has been proposed as a one-neutron halo candidate $[3,54]$. This hypothesis might be supported by recent $\gamma$-ray measurements, where the first $1^{-}$and $2^{-}$excited states of ${ }^{22} \mathrm{~N}$ were observed. Moreover, the first $3^{-}$state, which is a neutron unbound state, was measured in Ref. [55], where a significative 

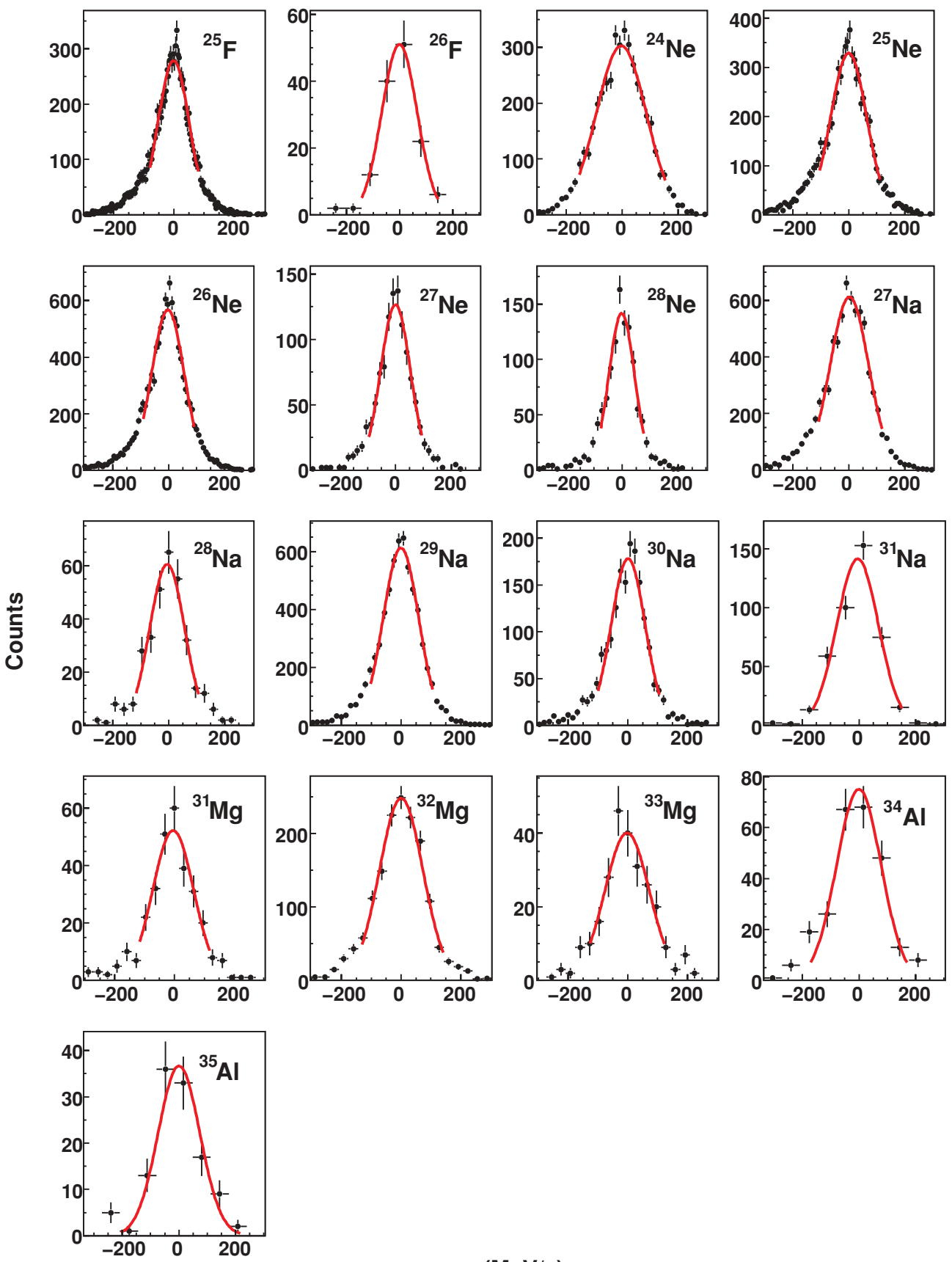

$$
\mathrm{p}_{\mathrm{z}}(\mathrm{MeV} / \mathrm{c})
$$

FIG. 5. (Color online) Fragment longitudinal-momentum distributions measured after one-neutron knockout of ${ }^{25,26} \mathrm{~F},{ }^{24-28} \mathrm{Ne},{ }^{27-31} \mathrm{Na}$, ${ }^{31-33} \mathrm{Mg}$, and ${ }^{34,35} \mathrm{Al}$ projectiles. The solid lines represent the Gaussian fits used to determine the widths of the distributions.

reduction of the $N=14$ shell gap, compared to the doubly magic nucleus ${ }^{22} \mathrm{O}$ is reported. This work provides the first one-neutron knockout data for ${ }^{22} \mathrm{~N}$, from which new structural information can be extracted. The results suggest a significant weight of the $1 s_{1 / 2}$ configuration.

Finally, ${ }^{26} \mathrm{~F}$, with $N=17$, also deserves a specific comment. The binding energy undergoes a significant reduction $\left(\mathrm{S}_{n}=1070 \pm 190 \mathrm{keV}\right.$ [41]) and the shell model predicts a rather stable ${ }^{25} \mathrm{~F}$ core, where the neutron levels are completely filled up to $1 s_{1 / 2}$ that is coupled to a single valence neutron in a $0 d_{3 / 2}$ orbit. Nevertheless, a previous one-neutron knockout experiment showed a low cross section and a narrow momentum distribution, with FWHM $=121 \pm 10 \mathrm{MeV} / \mathrm{c}$, that does not support such a configuration [44]. The authors interpreted these two facts as a possible indication of a different structure based on an excited ${ }^{25} \mathrm{~F}$ core and a $1 s_{1 / 2}$ valence neutron. The width obtained in this work is slightly higher, FWHM = $154 \pm 64 \mathrm{MeV} / \mathrm{c}$, but still compatible within the error bars. Neither did we observe any significant increase of the cross section, which is almost equal to the one measured for ${ }^{25} \mathrm{~F}$ projectiles. Both observations may suggest an important $1 s_{1 / 2}$ component, supporting the conclusions of Ref. [44]. 
TABLE I. Inclusive one-neutron knockout cross sections and widths of the fragment longitudinal-momentum distributions measured in this work. The widths of the momentum distributions obtained from other experiments are shown for comparison. The neutron number in the projectile $N$ and the one-neutron separation energy [41] $S_{n}$ are also included.

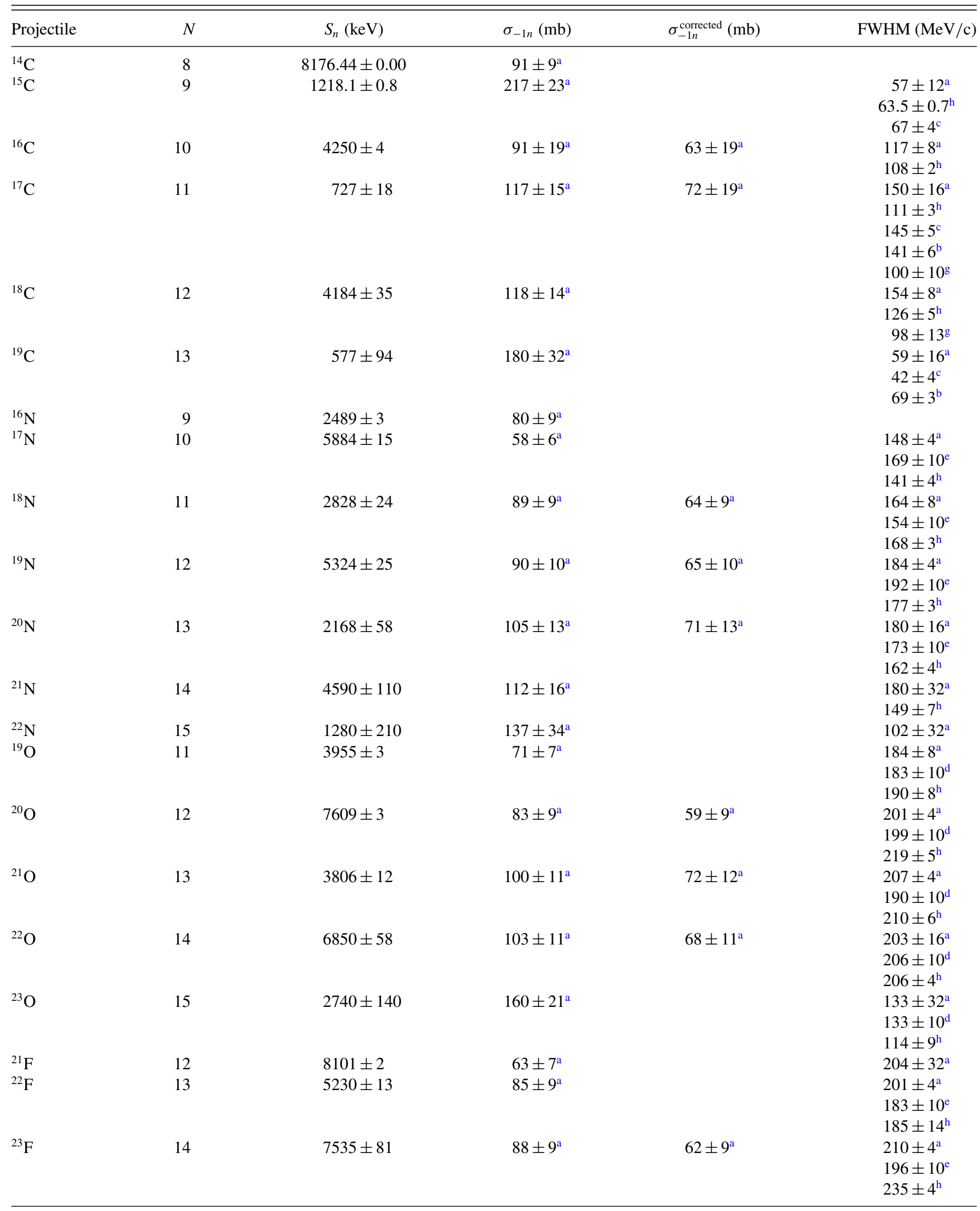


TABLE I. (Continued.)

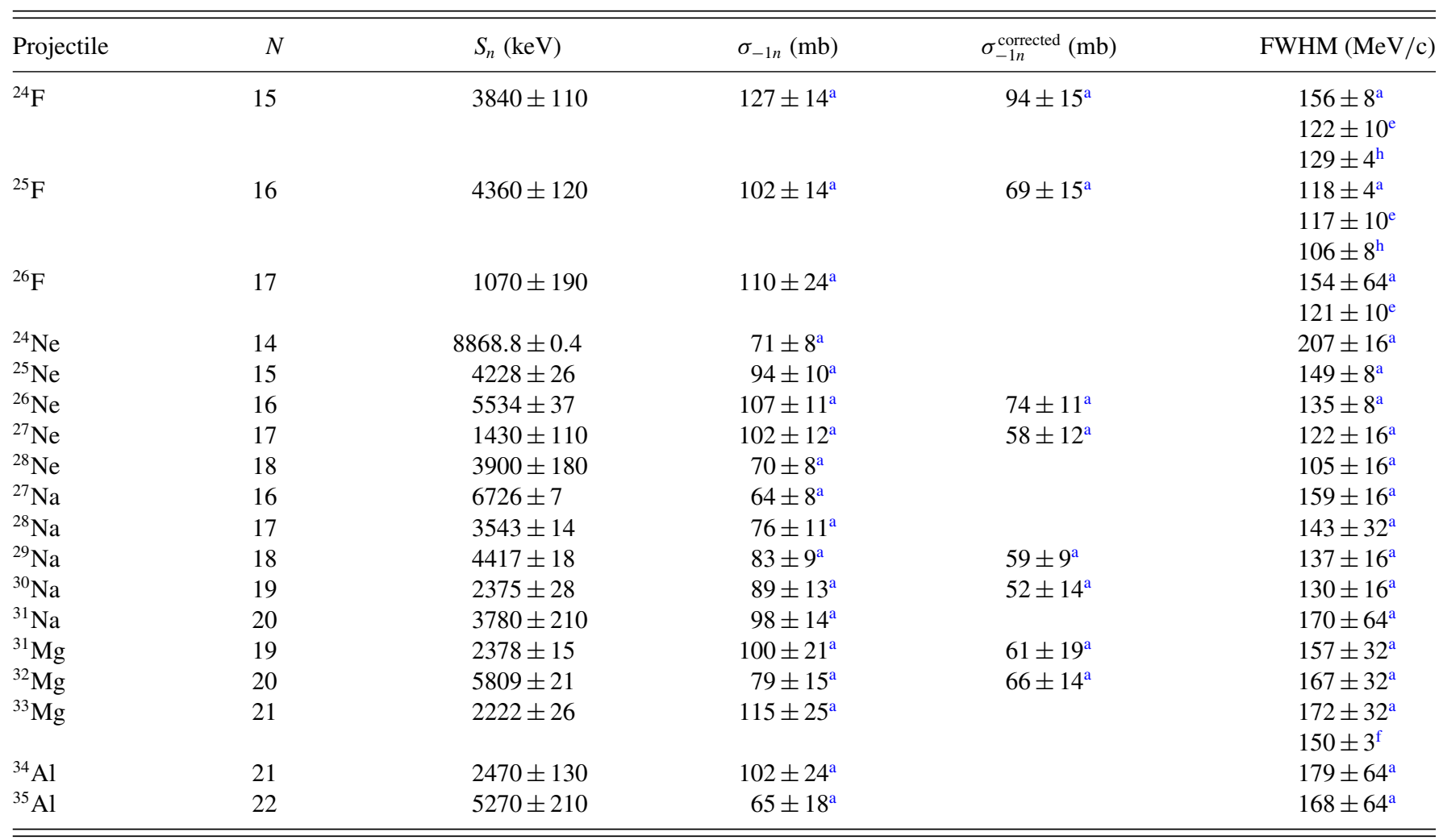

aThis work.

${ }^{\mathrm{b}}$ Baumann et al. [25].

${ }^{\mathrm{c} B a z i n}$ et al. [42].

${ }^{\mathrm{d}}$ Cortina et al. [43].

${ }^{e}$ Fernández Ph.D. dissertation [44].

${ }^{\mathrm{f}}$ Kanungo et al. [45].

${ }^{g}$ Ozawa et al. [46].

${ }^{\text {h}}$ Sauvan et al. [47].

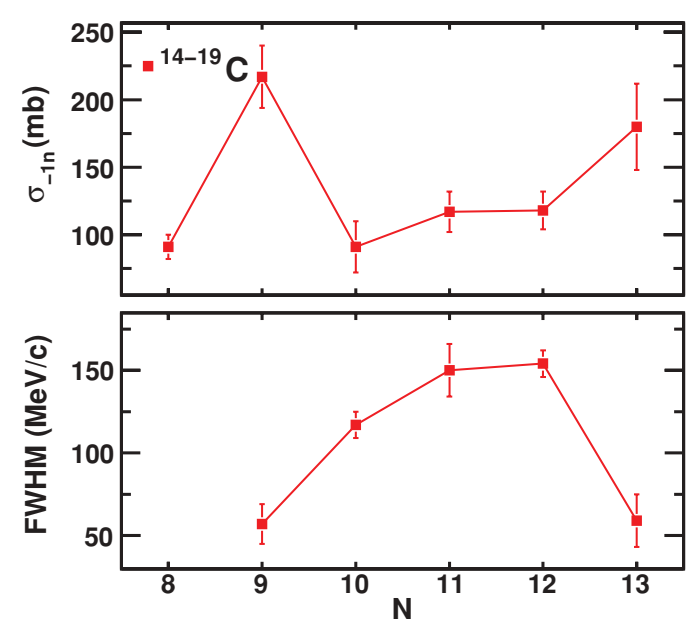

FIG. 6. (Color online) Inclusive results for ${ }^{14-19} \mathrm{C}$ projectiles. The one-neutron knockout cross sections and widths of the fragment longitudinal-momentum distributions are represented as a function of the neutron number in the projectile. The cross-section values are not corrected for reactions that occurred outside the target.

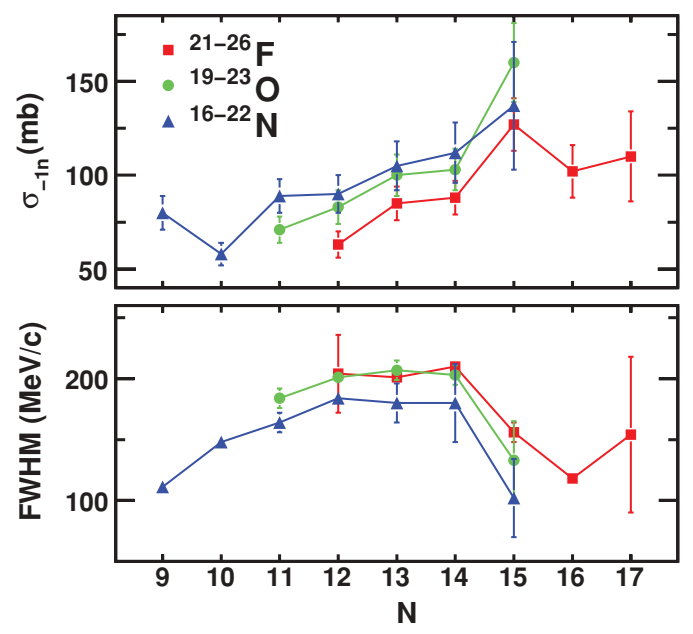

FIG. 7. (Color online) Inclusive results for ${ }^{16-22} \mathrm{~N},{ }^{19-23} \mathrm{O}$, and ${ }^{21-26} \mathrm{~F}$ projectiles. The one-neutron knockout cross sections and widths of the fragment longitudinal-momentum distributions are represented as a function of the neutron number in the projectile. The cross-section values are not corrected for reactions that occurred outside the target. 


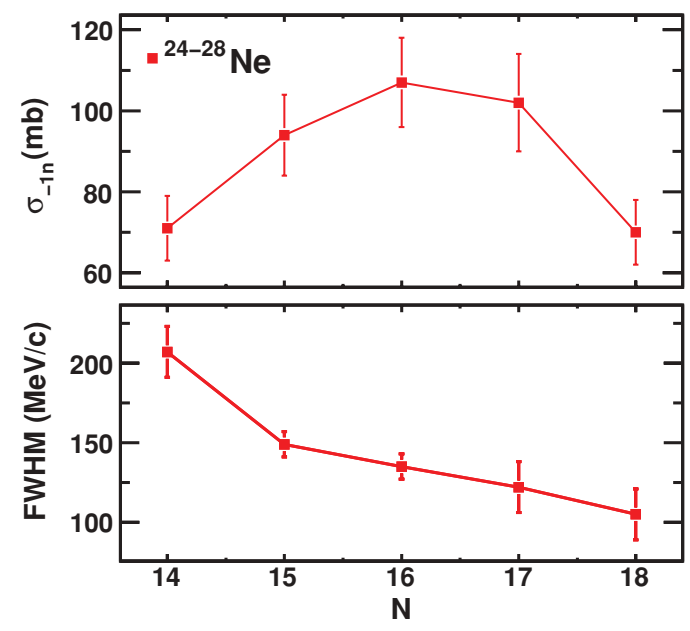

FIG. 8. (Color online) Inclusive results for ${ }^{24-28} \mathrm{Ne}$ projectiles. The one-neutron knockout cross sections and widths of the fragment longitudinal-momentum distributions are represented as a function of the neutron number in the projectile. The cross-section values are not corrected for reactions that occurred outside the target.

\section{Results for ${ }^{24-28} \mathrm{Ne}$}

According to a single-particle description within the conventional shell model, the last neutron of ${ }^{24-28} \mathrm{Ne}$ projectiles would occupy different orbits: ${ }^{24} \mathrm{Ne}\left(0 d_{5 / 2}\right),{ }^{25,26} \mathrm{Ne}\left(1 s_{1 / 2}\right)$ and ${ }^{27,28} \mathrm{Ne}\left(0 d_{3 / 2}\right)$. The validity of this simple picture can be investigated through the present results, summarized in Fig. 8. A reduction in the width of the fragment momentum distributions is found for projectiles above $N=14$, corresponding to the change from a $0 d_{5 / 2}$ to a $1 s_{1 / 2}$ configuration expected at this neutron number. The filling of the $0 d_{3 / 2}$ neutron orbit, predicted at $N=16$, should enlarge the width of the measured momentum distributions. However, the behavior of ${ }^{27,28} \mathrm{Ne}$ isotopes clearly contradicts this hypothesis and suggests significant contributions from $s_{1 / 2}$ neutrons. Further study of the experimental results presented here was carried out in Ref. [22], on the basis of a simple theoretical model that describes the longitudinal-momentum distributions as a function of the orbital angular momentum of the removed neutron, its separation energy, and a lower cutoff on the impact parameter that ensures the core survival. The conclusions obtained from this description clearly indicate that the ground states of ${ }^{27,28} \mathrm{Ne}$ are dominated by core excitations, with significant contributions from $s_{1 / 2}$ neutrons, and support the picture of ${ }^{28} \mathrm{Ne}$ proposed in Ref. [20], where exclusive oneneutron knockout data are analyzed.

\section{SUMMARY AND CONCLUSION}

One-neutron knockout reactions were used in this work to explore the evolution of nuclear structure near the neutron-drip line. Inclusive measurements of one-neutron knockout cross sections and fragment longitudinal-momentum distributions were carried out for 39 neutron-rich isotopes, ranging from carbon to aluminium and with neutron numbers from $N=8$ to 22 . These physical observables are sensitive to relevant structural phenomena such as neutron halos and can also be used to distinguish between $l=0$ and $l=2$ configurations in the $s d$ region.

The appearance of one-neutron halo structures was discussed for the odd-mass carbon isotopes ${ }^{15,17,19} \mathrm{C}$. In the case of ${ }^{15} \mathrm{C}$, indications of an extended spatial distribution of the valence neutron were found. However, the total-interaction cross-section measurements available in the literature do not seem to support this hypothesis, which should then be considered cautiously. The results obtained for ${ }^{17} \mathrm{C}$ show a clear halo suppression in this nucleus, caused by the predominance of a ${ }^{16} \mathrm{C} \otimes 0 d_{5 / 2}$ component in its ground state. Additionally, this work provides a new confirmation of a well-developed one-neutron halo in ${ }^{19} \mathrm{C}$.

Measurements carried out for nitrogen, oxygen, and fluorine isotopes reflect the change from $0 d_{5 / 2}$ to $1 s_{1 / 2}$ neutron configurations expected above $N=14$. In particular, this work provides the first one-neutron knockout data for ${ }^{22} \mathrm{~N}$, showing a significant $1 s_{1 / 2}$ admixture in its ground state. Results obtained for ${ }^{26} \mathrm{~F}$ might indicate a dominant configuration based on an excited state of the core coupled to a $1 s_{1 / 2}$ neutron that needs to be studied in more detail.

Neon isotopes also exhibit significant $1 s_{1 / 2}$ admixtures from $N=15$ to 16 . Above $N=16$, the ground state of ${ }^{27,28} \mathrm{Ne}$ seems to be dominated by excited cores that are coupled to valence neutrons in a $1 s_{1 / 2}$ orbital rather than $0 d_{3 / 2}$.

In general terms, the results presented in this work agree with previous one-neutron knockout experiments, which mainly studied species up to fluorine. They can be used to obtain a more solid picture of the ground-state structure for numerous nuclei and provide experimental data in a more extended region, reaching neutron-rich neon, sodium, magnesium, and aluminium isotopes.

\section{ACKNOWLEDGMENTS}

This work was supported by: GSI, via Hochschulzusammenarbeitsvereinbarungen under contracts DA RICK, OF ELZ, MZ KRAK; German BMBF; the DFG cluster of excellence Origin and Structure of the Universe; FBR under Contract No. 08-02-012244; EC under Contract No. ERBCHGE-CT92003. The Swedish Research Council and the Knut Alice Wallenberg Foundation; Ministerio de Ciencia e Innovación under Project Nos. FPA2007-62681 and FPA2007-62170; Ministerio de Educación under Grant No. FPU-AP2005-3308 and the Galician Consellería de Educación e Ordenación Universitaria, Grupos de Referencia Competitiva 2006/46.
[1] P. G. Hansen and B. Jonson, Europhys. Lett. 4, 409 (1987).

[2] B. Jonson, Phys. Rep. 389, 1 (2004).

[3] A. Ozawa, T. Kobayashi, T. Suzuki, K. Yoshida, and I. Tanihata, Phys. Rev. Lett. 84, 5493 (2000).
[4] D. Sohler et al., Phys. Rev. C 77, 044303 (2008).

[5] H. Iwasaki et al., Phys. Lett. B 491, 8 (2000).

[6] H. Iwasaki et al., Phys. Lett. B 481, 7 (2000).

[7] Z. Elekes et al., Phys. Rev. Lett. 98, 102502 (2007). 
[8] C. R. Hoffman et al., Phys. Rev. Lett. 100, 152502 (2008).

[9] C. R. Hoffman et al. Phys. Lett. B 672, 17 (2009).

[10] R. Kanungo et al., Phys. Rev. Lett. 102, 152501 (2009).

[11] A. Schiller et al., Phys. Rev. Lett. 99, 112501 (2007).

[12] M. Stanoiu et al., Phys. Rev. C 69, 034312 (2004).

[13] C. Thibault et al., Phys. Rev. C 12, 644 (1975).

[14] E. K. Warburton, J. A. Becker, and B. A. Brown, Phys. Rev. C 41, 1147 (1990).

[15] T. Motobayashi et al., Phys. Lett. B 346, 9 (1995).

[16] Y. Yanagisawa et al., Phys. Lett. B 566, 84 (2003).

[17] Y. Utsuno, T. Otsuka, T. Mizusaki, and M. Honma, Phys. Rev. C 60, 054315 (1999).

[18] Z. Dombrádi et al., Phys. Rev. Lett. 96, 182501 (2006).

[19] A. Obertelli et al., Phys. Rev. C 74, 064305 (2006).

[20] J. R. Terry et al., Phys. Lett. B 640, 86 (2006).

[21] M. Belleguic et al., Phys. Rev. C 72, 054316 (2005).

[22] C. Rodríguez-Tajes et al., Phys. Lett. B 687, 26 (2010).

[23] R. Anne et al., Nucl. Phys. A 575, 125 (1994).

[24] T. Aumann et al., Phys. Rev. Lett. 84, 35 (2000).

[25] T. Baumann et al., Phys. Lett. B 439, 256 (1998).

[26] D. Cortina-Gil et al., Phys. Lett. B 529, 36 (2002).

[27] V. Maddalena et al., Phys. Rev. C 63, 024613 (2001).

[28] N. Orr et al., Phys. Rev. Lett. 69, 2050 (1992).

[29] M. Smedberg et al., Phys. Lett. B 452, 1 (1999).

[30] P. G. Hansen and J. A. Tostevin, Annu. Rev. Nucl. Part. Sci. 53, 219 (2003)

[31] R. Serber, Phys. Rev. 72, 1008 (1947).

[32] H. Geissel et al., Nucl. Instrum. Methods B 70, 286 (1992).
[33] [http://www.gsi.de].

[34] M. Pfützner et al., Nucl. Instrum. Methods B 86, 213 (1994).

[35] V. Hlinka et al., Nucl. Instrum. Methods A 419, 503 (1998).

[36] N. Iwasa et al., Nucl. Instrum. Methods B 126, 284 (1997).

[37] C. A. Bertulani and K. W. McVoy, Phys. Rev. C 46, 2638 (1992).

[38] P. G. Hansen, A. S. Jensen, and B. Jonson, Annu. Rev. Nucl. Part. Sci. 45, 591 (1995).

[39] P. G. Hansen, Phys. Rev. Lett. 77, 1016 (1996).

[40] M. Smedberg, Ph.D. thesis, Chalmers tekniska höskola och Göteborgs universitet, 1998.

[41] G. Audi, A. H. Wapstra, and C. Thibault, Nucl. Phys. A 729, 337 (2003).

[42] D. Bazin et al., Phys. Rev. C 57, 2156 (1998).

[43] D. Cortina-Gil et al., Phys. Rev. Lett. 93, 062501 (2004).

[44] J. Fernández-Vázquez, Ph.D. thesis, Universidade de Santiago de Compostela, 2003.

[45] R. Kanungo et al., Phys. Lett. B 685, 253 (2010).

[46] A. Ozawa et al., Phys. Rev. C 78, 054313 (2008).

[47] E. Sauvan et al., Phys. Rev. C 69, 044603 (2004).

[48] D. Bazin et al., Phys. Rev. Lett. 74, 3569 (1995).

[49] E. Sauvan et al., Phys. Lett. B 491, 1 (2000).

[50] J. R. Terry et al., Phys. Rev. C 69, 054306 (2004).

[51] D. Cortina et al., Eur. Phys. J. A 10, 49 (2001).

[52] T. Nakamura et al., Phys. Rev. Lett. 83, 1112 (1999).

[53] E. C. Simpson and J. A. Tostevin, Phys. Rev. C 79, 024616 (2009).

[54] R. K. Gupta et al., J. Phys. G 26, L23 (2000).

[55] M. J. Strongman et al., Phys. Rev. C 80, 021302 (2009). 